\title{
Squamous cell and adenosquamous carcinomas of the gallbladder: clinicopathological analysis of 34 cases identified in 606 carcinomas
}

Juan C Roa ${ }^{1}$, Oscar Tapia ${ }^{1}$, Asli Cakir ${ }^{2}$, Olca Basturk ${ }^{3}$, Nevra Dursun ${ }^{4}$, Deniz Akdemir ${ }^{5}$, Burcu Saka ${ }^{6}$, Hector Losada ${ }^{7}$, Pelin Bagci ${ }^{8}$ and N Volkan Adsay

${ }^{1}$ Department of Pathology, Universidad de La Frontera, Temuco, Chile; ${ }^{2}$ Department of Pathology, Corlu State Hospital, Çorlu, Turkey; ${ }^{3}$ Department of Pathology, Memorial Sloan-Kettering Cancer Center, New York, NY, USA; ${ }^{4}$ Department of Pathology, Istanbul Education and Research Hospital, Istanbul, Turkey; ${ }^{5}$ Department of Biostatistics, Ohio Northern University, Ada, OH, USA; ${ }^{6}$ Department of Pathology, Gazi University, Ankara, Turkey; ${ }^{7}$ Department of Surgery, Universidad de La Frontera, Temuco, Chile and ${ }^{8}$ Department of Pathology, Emory University Hospital, Atlanta, GA, USA

The information in the literature on squamous cell and adenosquamous carcinomas of the gallbladder is highly limited. In this study, 606 resected invasive gallbladder carcinoma cases were analyzed. Squamous differentiation was identified in 41 cases $(7 \%)$. Those without any identifiable glandular-type invasive component were classified as pure squamous cell carcinomas (8 cases) and those with the squamous component constituting $25-99 \%$ of the tumors were classified as adenosquamous carcinomas (26 cases) and included into the analysis. The remaining 7 that had $<25 \%$ squamous component were classified as adenocarcinoma with focal squamous change and excluded. The clinicopathological characteristics of adenosquamous carcinoma/squamous cell carcinomas were documented and contrasted with that of ordinary gallbladder adenocarcinomas. The average patient age was 65 years (range 26-81); female/male ratio, 3.8. In only $13 \%$, there was a preoperative clinical suspicion of malignancy. Grossly, $58 \%$ presented as thickening and hardening of the wall and $6 \%$ were polypoid. In $12 \%$, mucosa adjacent to the tumor revealed squamous metaplasia. All pure squamous cell carcinomas had prominent keratinization. Giant cells and tumor-infiltrating eosinophils were observed in 29 and $51 \%$ of the squamous cell carcinomas/adenosquamous carcinomas versus $10 \%(P=0.02)$ and $6 \%(P=0.001)$ in gallbladder adenocarcinomas, respectively. All but three cases had 'advanced' (pT2 and above) carcinomas. Follow-up was available in 31 patients: 25 died of disease (median $=5$ months, range 0-20), and 6 were alive (median $=64$ months, range 5-112.5). The survival of patients with squamous cell carcinomas/adenosquamous carcinomas was significantly worse than that of gallbladder adenocarcinomas $(P=\mathbf{0 . 0 0 3})$, and this adverse prognosis persisted when compared with stage-matched advanced gallbladder adenocarcinoma cases (median $=11.4$ months, $P=0.01$ ). In conclusion, squamous differentiation was noted in $7 \%$ of gallbladder carcinomas. The incidence of adenosquamous carcinoma (defined as $25-99 \%$ of the tumor being squamous) was $4 \%$, and that of pure squamous cell carcinoma (without any documented invasive glandular component) was $1 \%$. Pure squamous cell carcinomas often showed prominent keratinization. The overall prognosis of adenosquamous carcinoma/squamous cell carcinoma appears to be even worse than that of ordinary adenocarcinomas. Most patients died within a few months; however, those few who were alive beyond 2 years in this cohort experienced long-term survival. Modern Pathology (2011) 24, 1069-1078; doi:10.1038/modpathol.2011.68; published online 29 April 2011

Keywords: adenosquamous; carcinoma; gallbladder; squamous

Correspondence: Professor NV Adsay, MD, Department of Pathology and Laboratory Medicine, Emory University Hospital, 1364 Clifton Road NE, Room H-180B, Atlanta, GA 30322, USA.

E-mail: volkan.adsay@emory.edu

This study was presented in part at the annual meeting of the United States and Canadian Academy of Pathology in Washington, DC, in 2010.

Received 27 October 2010; revised 2 March 2011; accepted 2 March 2011; published online 29 April 2011 
Gallbladder cancer is the most frequent neoplasm of the biliary tract with a clear predominance in the female population (2-6 times more frequent than in men). ${ }^{1-4}$ Its prevalence is widely variable, depending on the population studied: for example, in the United States, its frequency is $1.43 / 100000$ inhabitants, whereas in countries like Chile, this figure climbs up to 17.8/100000 inhabitants. ${ }^{3,5,6}$ At the same time, variations in its prevalence have been reported for both different indigenous groups and different geographical areas in the same country; facts that suggest important genetic and environmental influences on the development of the disease.

Adenocarcinomas are by far the most frequent histological subtype of the malignant gallbladder neoplasms, representing approximately $90-95 \%$ of all cases. ${ }^{1}$ In contrast, squamous cell or 'epidermoid' carcinomas and adenosquamous carcinomas are rare. ${ }^{1,7,8}$ The literature on these histologic types has been highly limited, mostly represented as individual case reports or analysis of small case series of a handful of cases. ${ }^{9-20}$ Furthermore, there has been no uniform definition for these tumors, with different authors employing different criteria: some regarded any squamous differentiation as a qualification for adenosquamous carcinoma even if it is a very small component of the tumor, and at the same time, others seemed to have included cases with substantial adenocarcinoma component into squamous cell carcinoma category. ${ }^{9,13,14,21-23}$ These definitional differences may partly be responsible for the variations in reported incidence of these tumors, which ranges from 1 to $12 \% .{ }^{14,18,24-28}$ Although the limited nature of the data and the definitional variations precluded proper characterization of these tumors, some common threads could be observed: it had been speculated that adenosquamous carcinomas/ squamous cell carcinomas are at least as aggressive as ordinary gallbladder adenocarcinomas..$^{9,11,13-15,23,29}$ It has been observed that the squamous component of gallbladder carcinomas proliferates at a higher rate than the glandular component; ${ }^{14}$ however, despite their high proliferation rate, these tumors appeared to less frequently present with lymph node metastasis than gallbladder adenocarcinomas. ${ }^{21}$ Their aggressive biological behavior has been attributed to their potential for direct extension and early invasion into the liver and neighboring organs, such as the stomach, duodenum and transverse colon..$^{9,21,23,29,30}$

The biology of carcinomas with squamous differentiation is intriguing. When all organs and sites are considered together, one might conclude that squamous cell carcinomas in general have a better prognosis than adenocarcinomas, although others would argue that this is related to the more superficial nature of squamous cancers occurring in accessible sites like skin, head and neck, gynecologic and genitourinary tracts, which are amenable to early diagnosis and easier surgical intervention. ${ }^{31}$
On the other hand, even advanced squamous cell carcinomas of these sites appear to have somewhat better prognosis than adenocarcinomas. ${ }^{32}$ This might give the impression that squamous cell carcinomas are slow-growing neoplasia when compared with malignancies of glandular type. However, this certainly does not seem to hold true for 'metaplastic' type squamous cell and adenosquamous carcinomas arising in glandular organs where adenocarcinomas are far more common. For example, in the breast and the pancreas, carcinomas with squamous differentiation have been shown to be aggressive neoplasia with a prognosis even worse than that of conventional adenocarcinomas of these respective sites. ${ }^{33-36}$ Whether this is also valid for squamous cell carcinoma/adenosquamous carcinoma of gallbladder or not has yet to be proven.

The aims of this study were to: (1) determine the frequency of squamous differentiation in the gallbladder; (2) provide more specific definitions for adenosquamous carcinoma and squamous cell carcinoma; (3) elucidate the clinicopathological characteristics of these tumors defined by these more specific criteria; and (4) determine the prognoses of these tumors and compare them with that of gallbladder adenocarcinoma. For this purpose, 606 gallbladder carcinomas obtained from different geographic regions were analyzed in detail retrospectively.

\section{Materials and methods}

\section{Cases}

Pathology material (reports and slides) from 606 consecutive invasive carcinomas of gallbladder identified in the authors' institutional files were retrieved and evaluated. Of these, 471 were from a high-gallbladder cancer incidence region (Hernán Henríquez Aravena Hospita, Universite de la Frontera, Temuco, Chile) and 135 from North America (Wayne State University, Detroit, MI, USA, and Emory University, Atlanta, GA, USA) where gallbladder cancer incidence is relatively low. In each case, a detailed analysis of the tumor was performed to determine the amount of squamous and glandular components. An average of 12 tumor slides per case was evaluated. Pure squamous cell carcinomas were entirely submitted and evaluated.

\section{Definitions}

\section{Squamous differentiation}

In the invasive carcinomas, the presence of any areas with squamous features, as defined and well characterized in other organs, was recognized and recorded as squamous differentiation. None of the cases in this study had features that could be classified as 'adenoacanthoma', and none of the squamous areas had the features of benign squamoid 
morules that can be seen in other neoplasms including 'adenomas' of gallbladder. In all cases, the squamous cells exhibited either or both cytologic atypia and/or dyskeratosis characteristic of malignant squamous cells.

\section{Pure squamous cell carcinoma}

Those invasive carcinomas composed exclusively of squamous differentiation without any recognizable invasive glandular component were classified as pure squamous cell carcinomas. This definition is very similar to that employed in urinary bladder. Presence of glandular dysplasia/carcinoma in situ was not considered a criterion of exclusion, as long as there were no invasive glandular elements.

\section{Adenosquamous carcinoma}

If the squamous component of the tumor constituted $25-99 \%$ of the tumor, it was classified as adenosquamous carcinoma. The '25\%' cutoff was selected arbitrarily as has been done for mixed differentiation cancers of other organs including the pancreas, where 'mixed' carcinomas have been well defined. ${ }^{37}$

\section{Carcinoma with focal squamous change}

Cases in which the squamous component constituted $<25 \%$ of the tumor were placed in this category and excluded from the analysis.

\section{Histopathological Analysis}

After the study cohort was identified based on the definitions provided above, it was then further analyzed for other morphological patterns and variations including amount of keratin, clear cell change, sarcomatoid change, goblet cells, sebaceous differentiation, cyst formation and necrosis. Association with an intracholecystic papillary tubular neoplasm, if present, was also duly recorded. Intracholecystic papillary tubular neoplasm is an unifying term the authors employ ${ }^{38-40}$ for all the tumoral intraepithelial neoplasms that are $>1 \mathrm{~cm}$, occurring in the gallbladder including what World Health Organization ${ }^{1}$ refers as 'adenoma' and 'intracystic papillary neoplasm' (papillary adenomas, and adenocarcinomas). In other words, these are gallbladder counterparts of pancreatic intraductal papillary mucinous neoplasms, ${ }^{1}$ biliary intraductal papillary neoplasms, ${ }^{1}$ and intraampullary papillary tubular neoplasms. ${ }^{1,38-41}$

In addition, both the study group and a control group (composed of 50 cases randomly selected from the excluded cases without any squamous differentiation) were analyzed for the following parameters: histological grade (well, moderate or poor, as determined by conventional approach), vascular invasion, perineural invasion, tumor giant cells and the amount of 'tumor-infiltrating' inflammatory cells (eosinophils and lymphocytes) as defined in other studies; ${ }^{42-44}$ ie, those associated preferentially with the tumor (in the vicinity of the tumor, in between the tumor cells or immediately adjacent to tumor cell clusters). The inflammation was semiquantitatively scored as $0=$ none, $1=$ minimal, $2=$ moderate and $3=$ marked, and subsequently grouped for comparison purposes as negligible (0 and 1) and significant (2 and 3 ).

Where applicable, statistical analysis was performed using Student's $t$-test for comparison.

\section{Clinical Parameters}

Information on the patients' gender and age as well as the clinical outcome were obtained through surgical pathology reports, patient's charts, from patient's primary physicians or Surveillance Epidemiology End Results (SEER) database in the United States, and from the Civil Registry as well as the databases of death certificates in the IT Department of the Head Office of the South Araucanía Health Service, in Chile. The patients who died within the first 30 days of the postoperative period were excluded from the survival analysis.

\section{Survival Analysis}

The survival analysis was performed using the statistical software Epi-info 6.0 and Stata 9.0. An exploratory analysis was performed and descriptive statistics subsequently applied with calculation of averages and s.d., medians and extreme values for continuous variables, calculation of percentages for category variables and Kaplan-Meier actuarial survival curves. Then, analytical statistics was applied, using $t$-test and analysis of variance (ANOVA) for continuous variables, Pearson's $\chi^{2}$ and Fisher's exact test for category variables and a log-rank test (CoxMantel) for survival comparisons. In addition to the comparison with the entire gallbladder adenocarcinoma group, the survival of adenosquamous carcinoma/squamous cell carcinoma was also separately compared with the advanced (pT2, pT3 and pT4) subset of gallbladder adenocarcinoma cases in order to eliminate the potential bias due to early carcinomas (pT1), which are common in the Temuco population.

\section{Ethical Aspects}

Helsinki principles were observed in the conduct of this study. In addition, the confidentiality of each patient's data was assured via their codification. The studies were performed in accordance with the institutional review board requirements.

\section{Results}

\section{Incidence and Clinical Features}

Squamous differentiation was identified in 41 of 606 invasive gallbladder carcinomas (7\%). Of these, 26 
cases (4\%) were adenosquamous carcinomas (25$99 \%$ of the tumor was squamous), and 8 (1\%) were pure squamous cell carcinomas. The remaining 7 with $<25 \%$ squamous component were classified as adenocarcinomas with focal squamous change and excluded.

The patients were 27 females and 7 males (female/ male ratio was 3.8 , same as in gallbladder adenocarcinoma). Mean age of the patients was 65 years (versus 64 in gallbladder adenocarcinoma) with a range of 26-81 years. In only $13 \%$, there was a preoperative clinical suspicion of malignancy.

\section{Pathological Features}

\section{Macroscopic features}

The median and average tumor sizes were 2.5 and $3.1 \mathrm{~cm}$, respectively (versus 2.4 and 2.7 in the control group of gallbladder adenocarcinoma; $P=0.42$ ). In $58 \%$, the tumor was not apparent even under macroscopic examination; carcinoma formed plaque-like mural thickening and induration indistinguishable from cholecystitis. In the remainder, there were irregular and variable amounts of nodular arrangements (Figure 1). The mucosal aspect of the tumor was ulcerated and hemorrhagic in some cases. Two cases had friable papillary/polypoid projections (Figure 1) consistent with an intracholecystic papillary tubular component. Of the tumors, $39 \%$ were located in the fundus, $15 \%$ in the lower third, $15 \%$ in the body and $31 \%$ had invaded more than one gallbladder segment. The exact incidence of gallstones could not be determined because in many cases, the gallstones had been removed by the surgeons as requested by the patients and this occurrence was not documented (and could not be verified accurately).

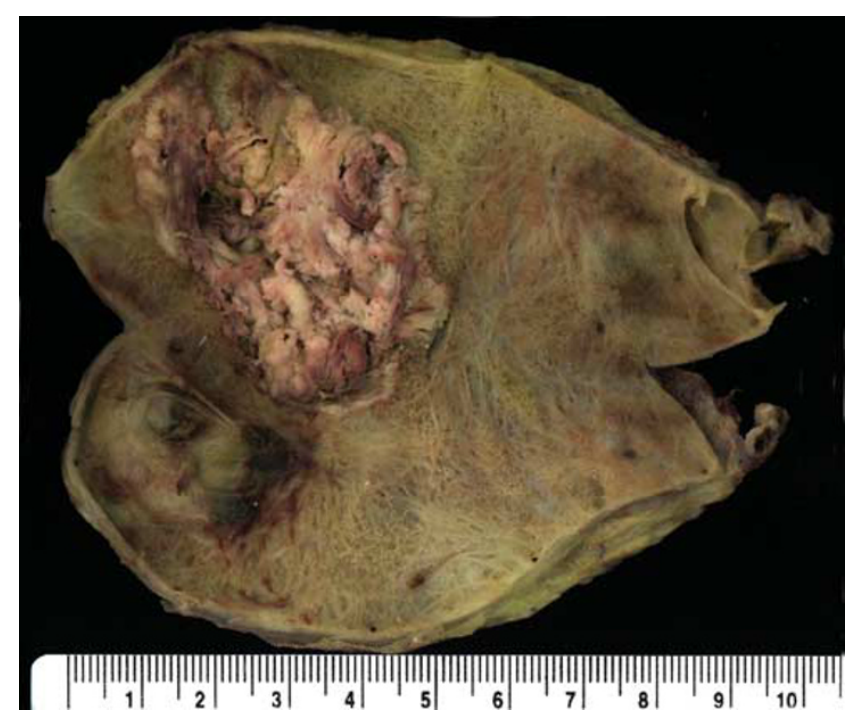

Figure 1 Adenosquamous carcinoma characterized with a broadbased ulcerofungating mass located in the fundus.

\section{Microscopic features}

A total of $65 \%(17 / 26)$ of ASCs had focal keratinization, whereas others were poorly differentiated; however, $88 \%$ (7/8) of pure squamous cell carcinomas had substantial keratinization including pearl formation and dyskeratotic cells (Figure 2). Four cases $(12 \%)$ revealed squamous metaplasia in the adjacent mucosa (Figure 3). Metaplastic foci had variable degrees of atypia. Six cases revealed focal sarcomatoid appearance with often pleomorphic spindle cells (Figure 4). Five displayed comedolike central necrosis in the large infiltrating squamous nests. Focal clear cell change was observed in five cases (Figure 5), and in one of these the tumor exhibited renal cell carcinoma-like pattern with alveolar growth and delicate vasculature. Interestingly, in three cases there was a peculiar microcystic change with accumulation of proteinaceouslike material within the cystic spaces (Figure 6).

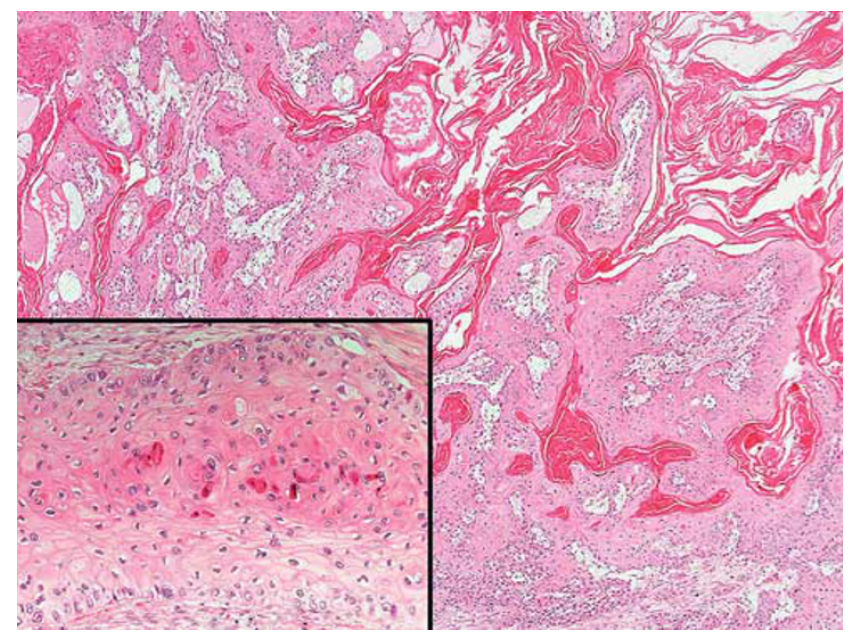

Figure 2 Pure squamous cell carcinoma with extensive keratinization including pearl formation and dyskeratotic cells (inset).

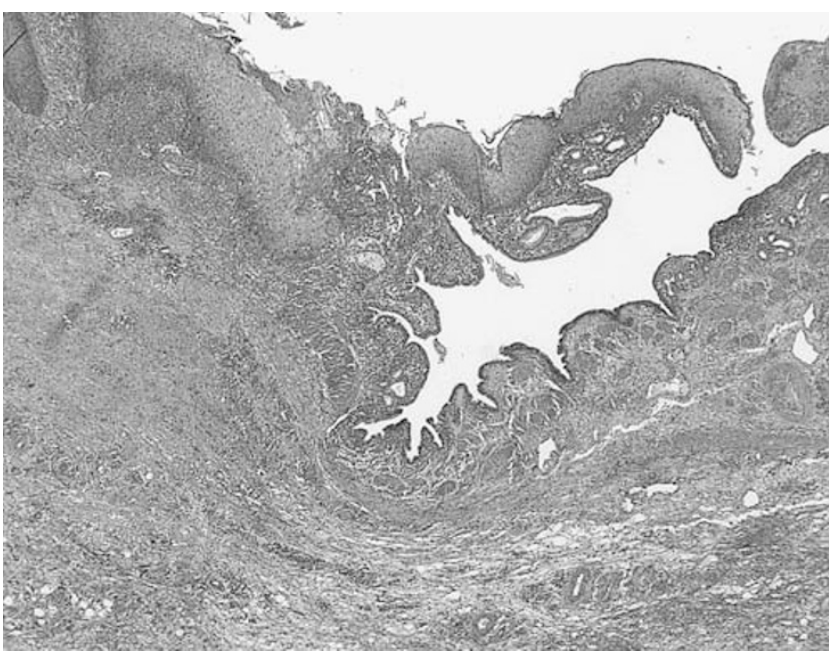

Figure 3 Adjacent gallbladder mucosa showing squamous metaplasia and/or dysplasia in four cases. 


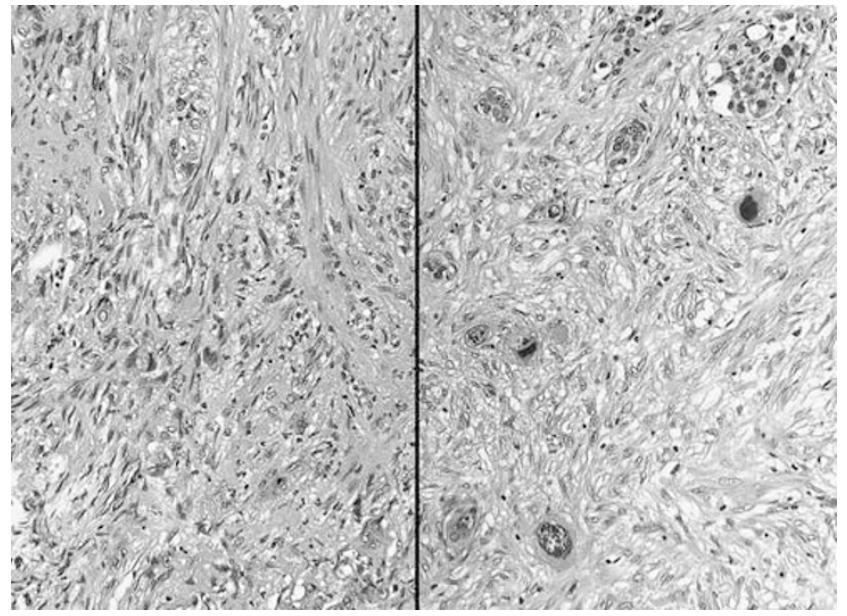

Figure 4 Predominant spindle cell component as well as bizarre and multinucleated giant tumor cells, focally imparting sarcomatoid appearance, are noted in six tumors.

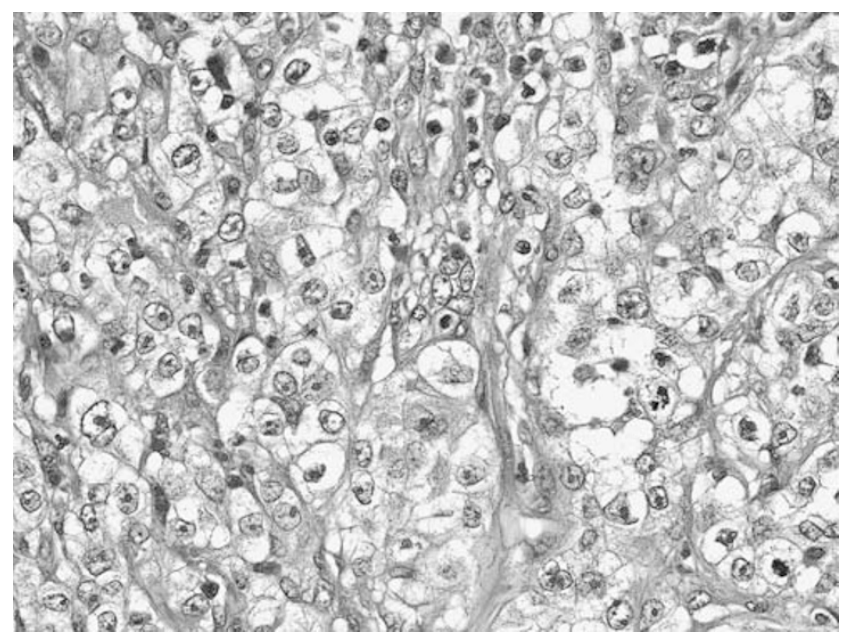

Figure 5 Four tumors reveal focal clear cell change characterized with optically clear cytoplasm and distinct cell borders, some mimicking renal cell carcinoma.

Sebaceous differentiation (Figure 7) and goblet cell change (Figure 8) were highly uncommon, each noted as focal findings in singular cases.

Invasive glandular components seen in the adenosquamous carcinoma cases displayed a spectrum of patterns similar to that seen in gallbladder adenocarcinomas.

In two cases, there was intracholecystic papillary tubular neoplasm. ${ }^{1,38-40}$ Squamous carcinoma cells were seen within the head of the polyp in these cases (Figure 9).

Bizarre, pleomorphic tumor giant cells (Figure 4) were identified in $29 \%$ of adenosquamous carcinomas/squamous cell carcinomas, whereas they were noted in only $10 \%$ of the gallbladder adenocarcinoma control group $(P=0.02)$.

Tumor-infiltrating eosinophils (Figure 10) were also more common in pure squamous cell

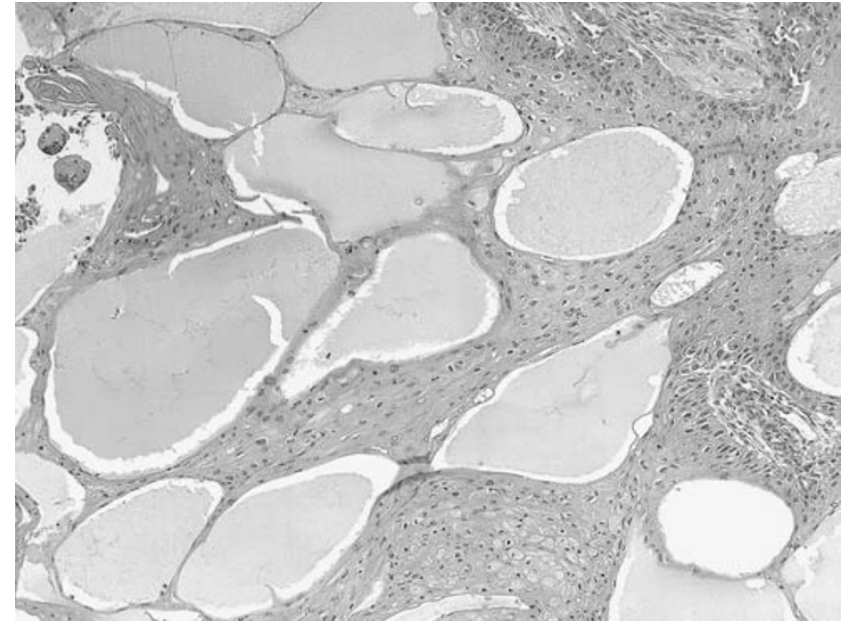

Figure 6 Microcysts filled with pale secretory material are seen in three cases.

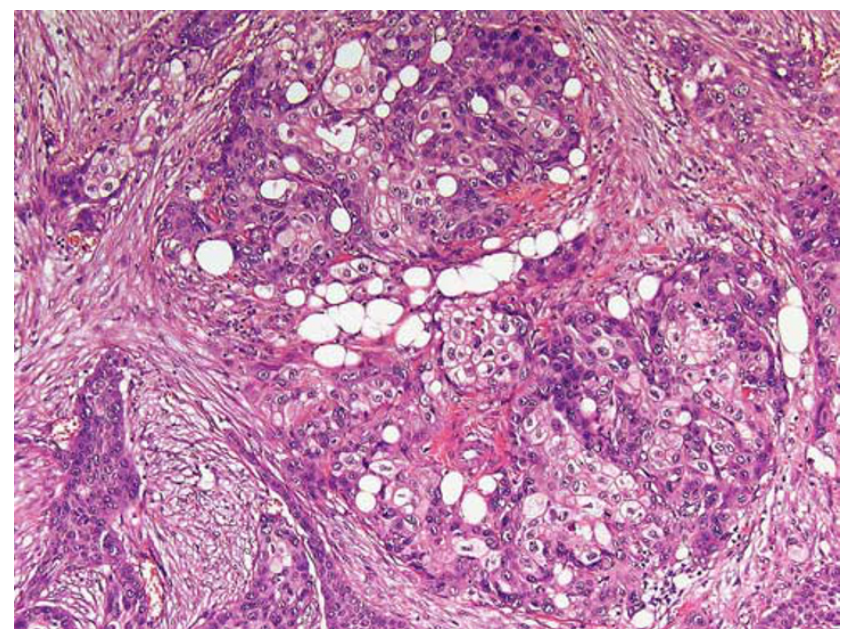

Figure 7 Sebaceous-like cells with bland nuclei and finely vacuolated cytoplasm-filled with lipid droplets-are noted within squamous nests of one adenosquamous carcinoma.

carcinomas $(51 \%$ had significant-score 2 and 3 -eosinophils) than in the gallbladder adenocarcinoma control group (only $6 \%$ had significant eosinophils; $P=0.001$ ). Tumor-infiltrating lymphocytes were also more common in pure squamous cell carcinomas than in the gallbladder adenocarcinoma control group; however, the difference was not significant (50 versus $34 \%$ ).

The incidence of vascular and perineural invasions was 76 and $32 \%$, respectively, versus 72 and $48 \%$ in gallbladder adenocarcinoma control group $(P=0.21$ and $P=0.22$, respectively). The majority were advanced carcinomas, most with invasion beyond the muscularis (T1b, 3 cases (9\%); T2, 8 cases $(24 \%)$; T3, 23 cases (68\%); T3: 23 cases $(68 \%)$ versus T3: 251 cases $(49 \%)$ in the gallbladder adenocarcinoma control group; $P=0.03)$. Also, $24 \%(8 / 34)$ had lymph node 


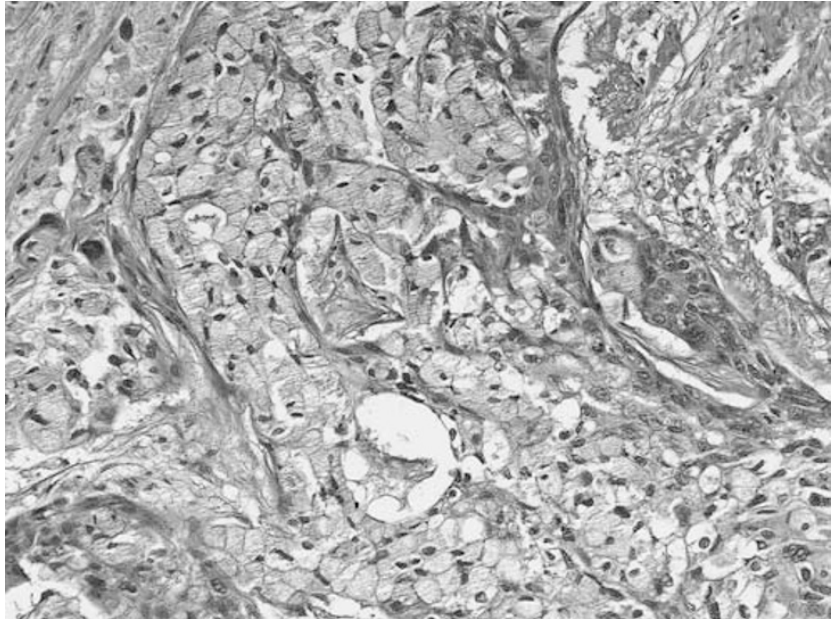

Figure 8 One adenosquamous carcinoma reveals a small focus with numerous goblet cells.

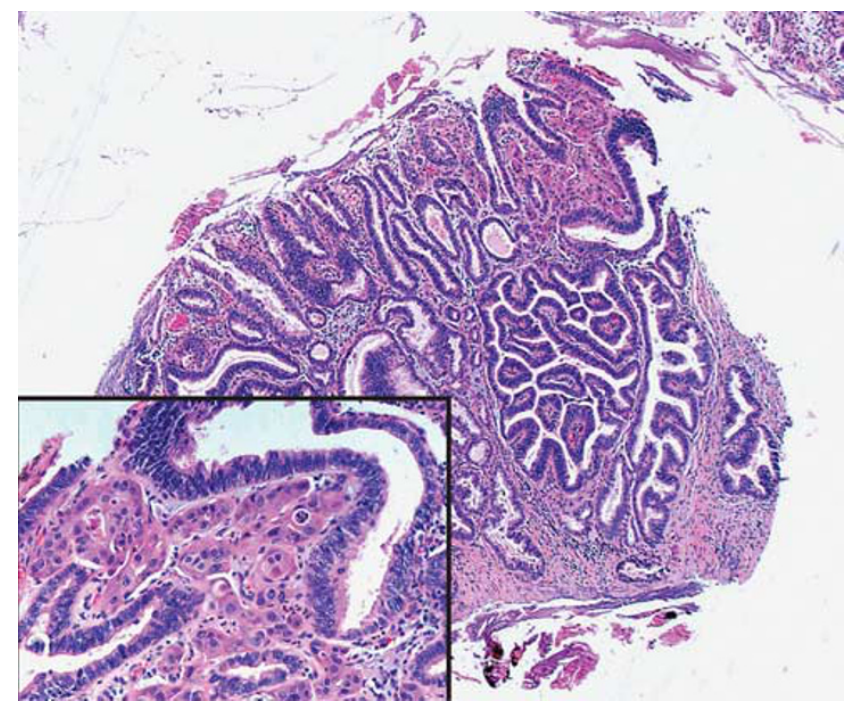

Figure 9 Mass-forming preinvasive component of adenosquamous carcinoma characterized with admixture of glandular and squamous foci.

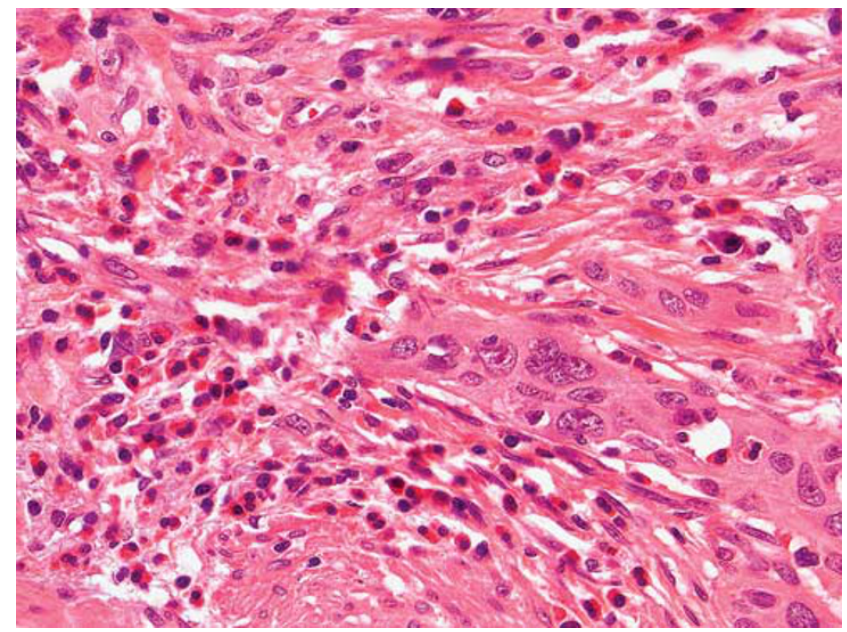

Figure 10 Pure squamous cell carcinomas are associated with peri/intratumoral eosinophilic infiltration.

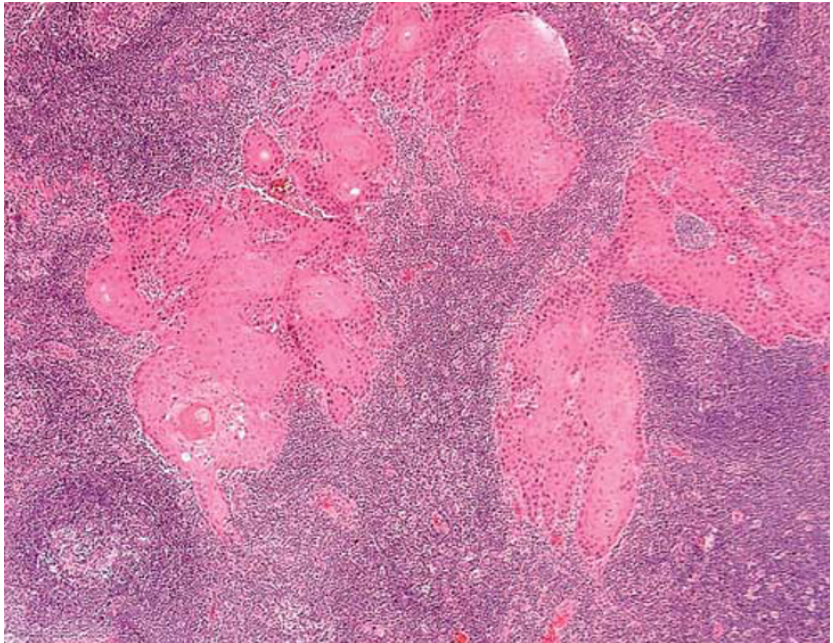

Figure 11 Five metastatic lymph nodes having squamous areas.

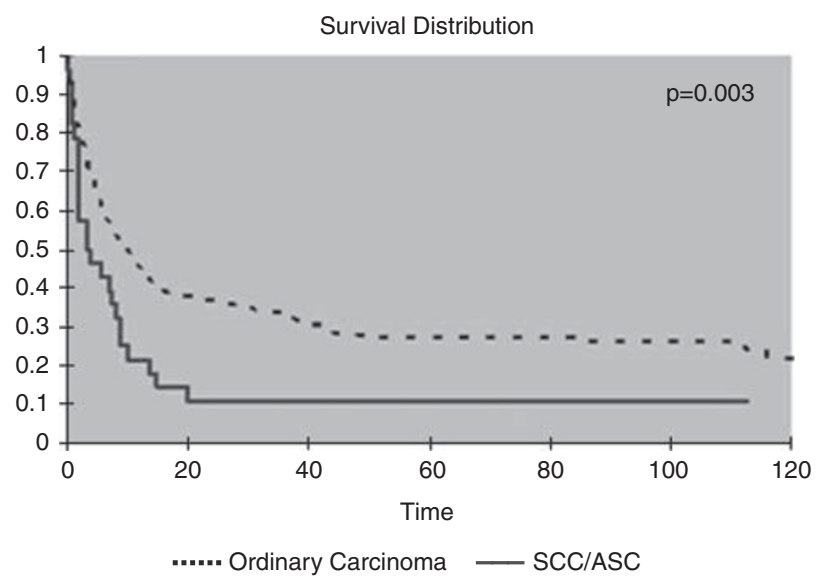

Figure 12 Kaplan-Meier survival curves comparing patients with pure squamous cell/adenosquamous carcinomas and those with ordinary carcinomas.

metastasis (6 adenosquamous carcinomas and 2 pure squamous cell carcinomas). Of seven lymph nodes available for our examination, five showed squamous areas (three ASCs and two SCCs; Figure 11).

\section{Clinical course}

Follow-up was available in 31 patients with adenosquamous carcinomas/squamous cell carcinomas, 25 of whom died of disease soon after diagnosis (median $=5$, range $1-20$ months) and 6 were alive (median $=64$, range 5-112.5 months). When compared with the all-comers of gallbladder adenocarcinomas, $(n=572)$ including the early (muscle confined) gallbladder cancers that are relatively common in the Temuco database, the survival of patients with squamous cell carcinomas/adenosquamous carcinomas was significantly worse than that 
Table 1 Comparison of (a) clinicopathological and (b) histopathological characteristics of squamous cell/adenosquamous carcinomas and ordinary adenocarcinomas of the gallbladder

(a)

\begin{tabular}{|c|c|c|c|}
\hline & $\begin{array}{c}\text { Adenosquamous carcinoma/ } \\
\text { squamous cell carcinoma }(\mathrm{N}=34)\end{array}$ & $\begin{array}{c}\text { Gallbladder } \\
\text { adenocarcinoma }(\mathrm{N}=572)\end{array}$ & $\mathrm{P}$-value \\
\hline Mean age & 65 & 64 & 0.59 \\
\hline Female/male & 3.8 & 3.8 & N/A \\
\hline Mean tumor size $(\mathrm{cm})$ & 3.1 & 2.7 & 0.42 \\
\hline Median tumor size $(\mathrm{cm})$ & 2.5 & 2.4 & N/A \\
\hline pT3 (\%) & 68 & 49 & 0.03 \\
\hline Mean survival (months) (range) & $23(1-112.5)$ & $50(1-160)$ & 0.003 \\
\hline \multirow{3}{*}{$\begin{array}{l}\text { Median survival (months) } \\
\text { (b) }\end{array}$} & 4 & 12 & \\
\hline & & & \\
\hline & $(\mathrm{N}=34)$ & $(\mathrm{N}=50)$ & \\
\hline Tumor giant cells (\%) & 29 & 10 & 0.02 \\
\hline Peritumoral eosinophils (\%) & 51 & 6 & 0.001 \\
\hline Vascular invasion (\%) & 76 & 72 & 0.21 \\
\hline Perineural invasion (\%) & 32 & 48 & 0.22 \\
\hline
\end{tabular}

For comparison of clinicopathologic parameters listed in table 1a, all 572 adenocarcinomas were used. For comparison of some histopathologic parameters that require more detailed analysis listed in table $1 \mathrm{~b}$, randomly chosen stage per stage matched 50 adenocarcinomas were used.

Table 2 Comparison of survival of squamous cell/adenosquamous carcinomas and ordinary adenocarcinomas of the gallbladder

\begin{tabular}{|c|c|c|c|}
\hline & $\begin{array}{l}\text { Adenosquamous carcinoma/squamous } \\
\text { cell carcinoma median survival (months) }\end{array}$ & $\begin{array}{l}\text { Gallbladder adenocarcinoma } \\
\text { median survival (months) }\end{array}$ & $\mathrm{P}$-value \\
\hline All stages & $\begin{array}{c}4 \\
(N=34)\end{array}$ & $\begin{array}{c}12 \\
(N=572)\end{array}$ & 0.003 \\
\hline Advanced stages (pT2, pT3, pT4) & $(N=31)$ & $\begin{array}{c}11.4 \\
(N=452)\end{array}$ & 0.001 \\
\hline pT2 stage & $\begin{array}{c}14,8 \\
(N=8)\end{array}$ & $\begin{array}{c}63 \\
(N=201)\end{array}$ & 0.01 \\
\hline pT3 stage & $\begin{array}{c}21.8 \\
(N=23)\end{array}$ & $\begin{array}{c}19.3 \\
(N=251)\end{array}$ & 0.09 \\
\hline
\end{tabular}

of gallbladder adenocarcinomas (median $=4$ vs 12 ; $P=0.003$; Figure 12). More importantly, this adverse prognosis persisted when compared with stage-matched 'advanced (pT2, pT3 and pT4)' gallbladder adenocarcinoma cases $(n=452)$ who had a median survival of 11.4 months $(P=0.01)$ as well as with individual stage groups with the exception of T3, which did not reach the same statistical significance $(P=0.09$, Table 2$)$.

The results are summarized in Tables 1 and 2 .

\section{Discussion}

Squamous differentiation is rather uncommon in the gallbladder. In this study of 606 invasive carcinomas, which represents the largest cohort to be analyzed for this purpose to date, squamous differentiation was detected in $7 \%$ of the cases. This figure varied significantly in the literature, ranging from 1 to $12 \%,{ }^{24-27}$ presumably mostly due to the definitional differences. Our study demonstrates that pure squamous cell carcinomas, which we defined as invasive carcinomas composed entirely of squamous differentiation, are very uncommon, constituting only $1 \%$ of the cases. Rather, squamous differentiation in the gallbladder is seen mostly as a secondary component in association with conventional adenocarcinomas. . $^{9,13,14,21,23,25,29,45,46}$ This focal squamous differentiation can vary in amount from very minimal, presumably with no (or negligible) clinical/biological significance, to extensive. We employed the arbitrary cutoff of ' $<25 \%$ ' to determine the negligibility of this secondary differentiation, as has been done for the mixed differentiation carcinomas of other organs, and classified such cases as adenocarcinoma with focal squamous change ( $1 \%$ of the cases), and reserved the diagnosis of adenosquamous carcinoma for only those that have $25-99 \%$ of the tumor showing squamous differentiation (4\% of gallbladder carcinomas). 
In order to determine the clinicopathological significance and associations of squamous differentiation in gallbladder carcinomas, we analyzed squamous cell carcinomas/adenosquamous carcinomas together and disregarded the cases with 'focal squamous change' from the analysis.

The clinical presentation of adenosquamous carcinoma/squamous cell carcinoma group in this study did not seem to be too different than ordinary gallbladder adenocarcinomas, and the impression in the literature is in accordance with this observation..$^{9,13-15,21,23,25,29}$ The patients are in their mid 60s-the youngest patient in this study was 26 -and it occurs predominantly in females $(\mathrm{F} / \mathrm{M}=3.8)$, similar to gallbladder adenocarcinomas (F/ $\mathrm{M}=3.8$ ). As is the case for most gallbladder carcinomas, preoperatively, most patients ( $>85 \%$ ) are not suspected to have cancer, and instead undergo cholecystectomy with the diagnosis of cholecystitis. The majority of the tumors $(\sim 40 \%)$ are located in the fundus, whereas the remainder involve the neck, body or the entire organ diffusely. The tumors appear to be slightly larger at presentation than ordinary gallbladder adenocarcinomas, but the difference was not statistically significant in this study (mean, 3.1 versus $2.7 ; P=0.42$ ).

Microscopically, one distinctive aspect of pure squamous cell carcinomas was that almost all (7/8) had substantial keratinization, showing abundant keratohyaline pearls, dyskeratotic cells and central deposition of dense keratin material within the infiltrative nests (Figure 2). Additionally, the presence of peritumoral eosinophils, which is a rather unusual and focal finding in otherwise ordinary gallbladder adenocarcinomas, was quite prominent in half of the squamous cell carcinomas. This may be interesting to note, because peritumoral eosinophilia (Figure 10) has been found to be rather common in squamous cell carcinomas, in particular, of the head and neck region, and some chemotactic factors and biologic/prognostic associations have also been implicated. ${ }^{47}$ Unfortunately, the number of cases were too small in this study to determine such similar association, if there was one, in gallbladder.

Although squamous cell carcinomas appeared to be rather well differentiated, with common and prominent keratinization (Figure 2), the squamous component of adenosquamous carcinomas were often of the poorly differentiated kind. In fact, adenosquamous carcinomas commonly displayed histologic signs of aggressiveness and high-grade features. Comedo-like necrosis (Figure 5) was noted in the infiltrative nests in multiple cases. More importantly, tumor giant cells (Figure 4), seen in a third of the cases, was significantly more common than in the control group $(P=0.02)$, and sarcomatoid change (Figure 4) was also seen in $20 \%$. This, however, may not be surprising as squamous differentiation in carcinomas of glandular organs often occur as a result of 'metaplastic' change, which often occurs as a part of 'dedifferentiation'. ${ }^{33,35,36,48}$
For example, in the breast, squamous differentiation is often a part of sarcomatoid carcinomas and highgrade transformation, and regarded under the category of 'metaplastic' carcinomas. ${ }^{36,48,49}$ On the other hand, although many gallbladder adenosquamous carcinomas/squamous cell carcinomas showed highgrade histologic features, it may also be important to note that focal aberrant differentiation toward other cell lineages such as sebaceous differentiation and goblet cells (Figures 7 and 8) were also occasionally encountered in these tumors. In fact, in some cases, a peculiar microcystic pattern of a well-differentiated carcinoma was exhibited (Figure 6).

Along with commonly higher-grade appearance especially of the adenosquamous carcinoma cases, the adenosquamous carcinoma/squamous cell carcinoma group also shows trends for being locally advanced (pT2, pT3 and pT4) carcinomas. The tumors were slightly larger in average size at presentation, although the difference did not reach statistical significance. More importantly, although two-thirds of adenosquamous carcinoma/squamous cell carcinoma cases were pT3 in our study, only half of the gallbladder adenocarcinomas were pT3s $(P=0.03)$, the rest were lower-stage tumors. That adenosquamous carcinomas/squamous cell carcinomas have a tendency to be more locally spread at diagnosis, especially to liver, has also been noted in the literature in some studies. ${ }^{9,21,23,29,30}$ On the other hand, some studies have noted (in very limited data) that although these tumors may have a higher tendency to invade to liver, the incidence of lymph node metastasis was lower than ordinary gallbladder adenocarcinomas. Unfortunately, we believe the data are too limited to determine this; the information in lymph node staging in our study was also not accurate enough to make this determination either.

The etiopathogenesis of adenosquamous carcino$\mathrm{ma} / \mathrm{squamous}$ cell carcinoma and whether it differs from that of gallbladder adenocarcinoma is difficult to determine. In general, chemical agents and irritants are known to induce squamous carcinogenesis, ${ }^{10,50}$ but although this has been established for organs that normally have squamous epithelium, the causes of squamous differentiation in carcinomas of glandular organs have been much less well understood, with the exception of the well-known parasitic association in urinary bladder. $^{51}$ In the gallbladder, some adenosquamous carcinomas/squamous cell carcinomas have been noted in association with parasitic infections as well,;2 however, most seem not to be related to parasites. Among our patients, none was known to have biliary flukes. It is conceivable that gallstones, which have a wellestablished role in gallbladder carcinogenesis, would possibly have a role in adenosquamous carcinomas/squamous cell carcinomas as well. Unfortunately, we do not have accurate information on the gallstone status of some of our patients, and therefore we cannot verify this association. No other known risk factors could be identified; our patients 
did not have primary sclerosing cholangitis or documented anomalous union or any syndromes that are known to be risk factors for gallbladder adenocarcinoma. In the literature, rare adenosquamous carcinomas/squamous cell carcinomas occurring in association with gallstones and parasitic infection have been documented, mostly as individual case reports, suggesting that this association may not be any different than that of ordinary gallbladder adenocarcinomas. ${ }^{8}$ It may be interesting to note that $\sim 12 \%$ of the cases in our study had squamous changes in the adjacent mucosa, indicating that whatever was the instigator was also presumably leading to early mucosal changes first, followed by the dysplasia-carcinoma sequence. Also, interestingly, two cases arose in association with a mass-forming preinvasive neoplasm, which we call intracholecystic papillary neoplasm. ${ }^{38}$ In our experience, this adenomacarcinoma pathway is responsible for $\sim 10 \%$ of the invasive carcinomas, ${ }^{38}$ and therefore it would not be surprising that 2 of the 34 adenosquamous carcinoma/squamous cell carcinoma cases also arose in association with this pathway.

Unlike the squamous cell carcinomas arising in normally squamous-lined organs, adenosquamous carcinomas/squamous cell carcinomas of gallbladder appear to be highly aggressive neoplasia, similar to those arising in other glandular organs such as pancreas and breast, with a prognosis at least as dismal, and possible even worse than that of the ordinary gallbladder adenocarcinoma. Most of our patients died within a few months, with a median survival of 5 months, which is incomparably worse than when all gallbladder adenocarcinomas are considered together (including those early carcinomas encountered in the Temuco population); however, it was also significantly more aggressive even when compared with the control group of randomly selected 'advanced (pT2, pT3 and pT4)' gallbladder adenocarcinoma cases (with median survival of 11.4 months; $P=0.003$ ). Although most patients died within a few months, those few who were alive beyond 2 years in this cohort intriguingly experienced long-term survival.

In summary, adenosquamous carcinomas/squamous cell carcinomas of gallbladder are uncommon, together constituting $\sim 5 \%$ of the malignancies of this organ. They are often fairly advanced (pT2, pT3 and pT4) cancers at the time of diagnosis, and their prognosis is dismal if not more so than that of ordinary gallbladder adenocarcinomas. More studies are needed to determine the etiopathogenetic and molecular differences, if any, of this histologic type compared with conventional adenocarcinomas.

\section{Acknowledgements}

This study is supported in part by the Georgia Cancer Coalition Distinguished Cancer Clinicians and Scientists Program and in part by the Office of
Research of the Universidad de la Frontera and Fondecyt Grant 1090171.

\section{Disclosure/conflict of interest}

The authors declare no conflict of interest.

\section{References}

1 Albores-Saavedra J, Klöppel G, Adsay NV, et al. Carcinoma of the Gallbladder and Extrahepatic Bile Ducts, 4th edn. WHO Press: Geneva, 2010.

2 Lazcano-Ponce EC, Miquel JF, Munoz N, et al. Epidemiology and molecular pathology of gallbladder cancer. CA Cancer J Clin 2001;51:349-364.

3 Randi G, Franceschi S, La Vecchia C. Gallbladder cancer worldwide: geographical distribution and risk factors. Int J Cancer 2006;118:1591-1602.

4 Roa I, Araya JC, Wistuba I, et al. [Gallbladder cancer in the IX Region of Chile. Impact of the anatomopathological study of 474 cases]. Rev Med Chil 1994;122: 1248-1256.

5 Henson DE, Schwartz AM, Nsouli H, et al. Carcinomas of the pancreas, gallbladder, extrahepatic bile ducts, and ampulla of vater share a field for carcinogenesis: a population-based study. Arch Pathol Lab Med 2009;133:67-71.

6 Medina E, Kaempffer AM. [Cancer mortality in Chile: epidemiological considerations]. Rev Med Chil 2001; 129:1195-1202.

7 Adsay NV, Klimstra DS. Benign and malignant tumors of the gallbladder and extrahepatic biliary tract. In: Odze RD, Goldblum JR (eds). Surgical Pathology of the GI Tract, Liver, Biliary Tract, and Pancreas. Elsevier: Philadelphia, 2009, pp 845-875.

8 Albores-Saavedra J, Henson DE, Klimstra DS. Gallbladder cancer. In: Rosai J (ed). Tumors of the Gallbladder, Extrahepatic Bile Ducts and Ampulla of Vater. Atlas of Tumor Pathology. AFIP: Washington, 2000, pp 37-106.

9 Chan KM, Yu MC, Lee WC, et al. Adenosquamous/ squamous cell carcinoma of the gallbladder. J Surg Oncol 2007;95:129-134.

10 Andrea C, Francesco C. Squamous-cell and nonsquamous-cell carcinomas of the gallbladder have different risk factors. Lancet Oncol 2003;4:393-394.

11 del Pozo AC, De Battista S, Velasco D, et al. [Epidermoid carcinoma of gallbladder: analysis of our casuistic]. Acta Gastroenterol Latinoam 2005;35:162-164.

12 Karasawa T, Itoh K, Komukai M, et al. Squamous cell carcinoma of gallbladder-report of two cases and review of literature. Acta Pathol Jpn 1981;31:299-308.

13 Mingoli A, Brachini G, Petroni R, et al. Squamous and adenosquamous cell carcinomas of the gallbladder. J Exp Clin Cancer Res 2005;24:143-150.

14 Nishihara K, Nagai E, Izumi Y, et al. Adenosquamous carcinoma of the gallbladder: a clinicopathological, immunohistochemical and flow-cytometric study of twenty cases. Jpn J Cancer Res 1994;85:389-399.

15 Waisberg J, Bromberg SH, Franco MI, et al. Squamous cell carcinoma of the gallbladder. Sao Paulo Med J 2001;119:43.

16 Gupta S, Gupta SK, Aryya NC. Primary squamous cell carcinoma of gallbladder presenting as acute cholecystitis. Indian J Pathol Microbiol 2004;47:231-233. 
17 Hanada M, Shimizu H, Takami M. Squamous cell carcinoma of the gallbladder associated with squamous metaplasia and adenocarcinoma in situ of the mucosal columnar epithelium. Acta Pathol Jpn 1986;36:1879-1886.

18 Ishikawa Y, Yoshida H, Mamada Y, et al. [Squamous cell carcinoma of gallbladder]. J Nippon Med Sch 2004;71:417-420.

19 Kobayashi A, Miyagawa S, Miwa S, et al. Radical surgery for advanced squamous cell carcinoma of the gallbladder: a report of three cases, including a 10-year survivor. Hepatogastroenterology 2007;54: 350-353.

20 Kumar A, Singh MK, Kapur BM. Synchronous double malignant tumors of the gall bladder. A case-report of squamous cell carcinoma with an angiosarcoma. Eur J Surg Oncol 1994;20:63-67.

21 Kondo M, Dono K, Sakon M, et al. Adenosquamous carcinoma of the gallbladder. Hepatogastroenterology 2002;49:1230-1234.

22 Kusakina GK, Nigmatulina AA. [Adenosquamous cancer of the gallbladder]. Arkh Patol 1980;42:57-59.

23 Lada PE, Taborda B, Sanchez M, et al. [Adenosquamous and squamous carcinoma of the gallbladder]. Cir Esp 2007;81:202-206.

24 Duffy A, Capanu M, Abou-Alfa GK, et al. Gallbladder cancer (GBC): 10-year experience at Memorial SloanKettering Cancer Centre (MSKCC). J Surg Oncol 2008;98:485-489.

25 de Aretxabala X, Roa I, Burgos L, et al. Gallbladder cancer: an analysis of a series of 139 patients with invasion restricted to the subserosal layer. J Gastrointest Surg 2006;10:186-192.

26 Sons HU, Borchard F, Joel BS. Carcinoma of the gallbladder: autopsy findings in 287 cases and review of the literature. J Surg Oncol 1985;28:199-206.

27 Henson DE, Albores-Saavedra J, Corle D. Carcinoma of the gallbladder. Histologic types, stage of disease, grade, and survival rates. Cancer 1992;70:1493-1497.

28 Zhang L, Chen Z, Fukuma M, et al. Prognostic significance of race and tumor size in carcinosarcoma of gallbladder: a meta-analysis of 68 cases. Int J Clin Exp Pathol 2008;1:75-83.

29 Oohashi Y, Shirai Y, Wakai T, et al. Adenosquamous carcinoma of the gallbladder warrants resection only if curative resection is feasible. Cancer 2002;94: 3000-3005.

30 Saito A, Noguchi Y, Doi C, et al. A case of primary adenosquamous/squamous cell carcinoma of gallbladder directly invaded duodenum. Hepatogastroenterology 1999;46:204-207.

31 Mendenhall WM, Wernig JW, Pfister DG. Cancer of head and neck. In: DeVita V, Lawrence TS, Rosenberg SA (eds). DeVita, Hellman, and Rosenberg's Cancer: Principles \& Practice of Oncology, 8th edn. Lippincott Williams and Wilkins: Philadelphia, 2008.

32 Barnes L, Brandwein M, (eds). Surgical Pathology of the Head and Neck. Marcel Decker Inc: New York, 2001.

33 Bossuyt V, Fadare O, Martel M, et al. Remarkably high frequency of EGFR expression in breast carcinomas with squamous differentiation. Int J Surg Pathol 2005; 13:319-327.

34 Kardon DE, Thompson LD, Przygodzki RM, et al. Adenosquamous carcinoma of the pancreas: a clinicopathologic series of 25 cases. Mod Pathol 2001;14: $443-451$.
35 Wargotz ES, Norris HJ. Metaplastic carcinomas and sarcomas of the breast. Am J Clin Pathol 1991;96:781.

36 Beatty JD, Atwood M, Tickman R, et al. Metaplastic breast cancer: clinical significance. Am J Surg 2006; 191:657-664.

37 Hruban RH, Pitman MB, Klimstra DS. Tumors of the pancreas. AFIP Atlas of Tumor Pathology, 4th series, Fascicle 6 edn American Registry of Pathology: Washington, DC, 2007.

38 Dursun N, Roa JC, Tapia O, et al. Intravesicular papillary-tubular neoplasm (IVPN) as a unifying category for mass forming preinvasive neoplasms of the gallbladder: an analysis of 87 cases. Mod Pathol 2010;23:144A.

39 Jang KT, Dursun N, Basturk O, et al. Immunohistochemical analysis of the progression of flat versus tumoral intraepithelial neoplasia (intracholecysitc papillary-tubular neoplasm) in gallbladder carcinogenesis. Mod Pathol 2011;24:363A.

40 Jang KT, Dursun N, Basturk O, et al. Classification of tumoral intraepithelial neoplasms of the gallbladder under a unified category of intracholecystic papillary tubular neoplasms (icpn) with 4 subsets discernible by correlation of morphology and immunophenotype. Mod Pathol 2011;24:363A.

41 Ohike N, Kim GE, Tajiri $\mathrm{T}$, et al. Intra-ampullary papillary-tubular neoplasm (IAPN): characterization of tumoral intraepithelial neoplasia occurring within the ampulla: a clinicopathologic analysis of 82 cases. Am J Surg Pathol 2010;34:1731-1748.

42 Alrawi SJ, Tan D, Stoler DL, et al. Tissue eosinophilic infiltration: a useful marker for assessing stromal invasion, survival and locoregional recurrence in head and neck squamous neoplasia. Cancer J 2005;11: 217-225.

43 Quaedvlieg PJ, Creytens DH, Epping GG, et al. Histopathological characteristics of metastasizing squamous cell carcinoma of the skin and lips. Histopathology 2006;49:256-264.

44 Ohashi Y, Ishibashi S, Suzuki T, et al. Significance of tumor associated tissue eosinophilia and other inflammatory cell infiltrate in early esophageal squamous cell carcinoma. Anticancer Res 2000;20:3025-3030.

45 Herrera-Goepfert R, Manrique Ortega JJ, RodriguezMartinez HA. Carcinosarcoma of the gallbladder. Histol Histopathol 1987;2:273-275.

46 Stamatiadis AN, Papadimitriou C, Zombolas BT, et al. [Adenosquamous carcinoma of the gallbladder]. Minerva Chir 1989;44:2085-2087.

47 Korkmaz H, Caydere M, Dursun E, et al. Prognostic importance of lymphatic reaction pattern in laryngeal carcinoma. Am J Otolaryngol 1999;20:298-303.

48 Rosen DD, (ed) Rosen's Breast Pathology. Lippincott Williams and Wilkins: Philadelphia, 2009, pp 470-515.

49 Wargotz ES, Deos PH, Norris HJ. Metaplastic carcinomas of the breast. II. Spindle cell carcinoma. Hum Pathol 1989;20:732-740.

50 Muto Y, Sho Y, Kurihara K, et al. [Morphological study of carcinoma of the gallbladder: its differences between calculous and acalculous carcinoma]. Nippon Geka Gakkai Zasshi 1985;86:846-852.

51 El Adl MM, Yamase HT, Nieh PT, et al. ABH cell surface isoantigens in invasive bladder carcinoma associated with schistosomiasis. J Urol 1984;131:249-251.

52 Gomez N, Urrea I, Astudillio R. Primary epidermoid carcinoma of the gallbladder. Acta Gastroenterol Latinoam 1990;20:169-173. 